\title{
Building a Bridge Between Primary and Perioperative Care: Addressing the Challenges of Perioperative Buprenorphine Maintenance and Postdischarge Therapy
}

\author{
Ivan Urits, MD, ${ }^{1}$ Omar Viswanath, MD, ${ }^{2,3,4}$ Julie Petro, MD, ${ }^{1}$ Cyrus Yazdi, MD ${ }^{1}$ \\ ${ }^{1}$ Department of Anesthesiology, Critical Care, and Pain Medicine, Beth Israel Deaconess Medical Center, Harvard Medical School, Boston, MA \\ ${ }^{2}$ Valley Anesthesiology and Pain Consultants, Phoenix, AZ ${ }^{3}$ University of Arizona College of Medicine-Phoenix, Phoenix, AZ \\ ${ }^{4}$ Creighton University School of Medicine, Omaha, NE
}

As awareness of the opioid use disorder epidemic has increased, the number of people on rehabilitative medicationassisted treatment has seen a concurrent rise. Buprenorphine, available in multiple preparations, plays an important role in opioid detoxification, addiction therapy, and chronic pain management. Cizdol, Subutex, Suboxone, Zubsolv, and Bunavail are available as buprenorphine hydrochloride $(\mathrm{HCl})$ alone or as a combination of buprenorphine and naloxone $\mathrm{HCl}$ and are typically used to treat opioid addiction. Temgesic is available as a sublingual tablet to treat moderate to severe pain. Buprenex is formulated as a solution for injection and is often used for acute pain in primary care settings. Norspan and Butrans are transdermal preparations used for management of chronic pain. ${ }^{1}$

Buprenorphine is a semisynthetic opioid that is 30 times more potent than morphine and acts as a partial agonist at the mu receptor and as an antagonist at the kappa receptor. Because of these pharmacologic properties, buprenorphine binds strongly to opioid receptors, displacing and preventing the binding of other opioid drugs that possess lower receptor affinity although higher intrinsic activity. As a result, buprenorphine use by patients undergoing surgery poses a unique challenge to clinicians. The pharmacologic properties of buprenorphine make achieving adequate pain relief difficult, especially during the potentially tumultuous perioperative period. Surgical patients who are maintained on buprenorphine often require unusually high opioid doses to overcome buprenorphine receptor binding and achieve adequate analgesia.

With the increasing prevalence of buprenorphine in the general patient population coupled with the fact that these same patients are now presenting for more surgeries, both primary care and perioperative care clinicians must quickly become adept at understanding the complex interactions and preparations of this drug. Surgery presents numerous inherent risks for patients who are on long-term buprenorphine therapy, and no guidelines are available to enable primary and perioperative clinicians to agree on how best to bring their mutual patient into, through, and even more important, out of the perioperative period and back to a maintenance regimen.

Various strategies have been reported to help guide perioperative management of these patients. Briefly, buprenorphine management decision-making can be based on the pain expected from the planned procedure. ${ }^{2}$ Patients undergoing low pain risk procedures may benefit from maintaining their buprenorphine regimen. For these patients, the partial agonist effect of buprenorphine may provide sufficient analgesic effect, so minimal additional opioids may be required to adequately manage pain. In contrast, patients undergoing high pain risk procedures may benefit from a preoperative bridge to pure opioid therapy for the perioperative period. ${ }^{3}$ For these patients, we can assume that the systemic presence of buprenorphine would limit the effectiveness of additionally administered opioid drugs, and patients would thus require exceptionally high doses of additional breakthrough opioid medication.

Despite multiple reported guidelines, however, we have no generalized consensus for management given the multitude of patient variables and the complexity of buprenorphine management. Furthermore, patients managed on buprenorphine may not already be an established pain clinic patient, thereby increasing the burden of medical decision-making prior to the perioperative period on the primary care physician who must make an informed decision from nonuniform guidelines.

Figures 1 and 2 show the guidelines used at our institution, Beth Israel Deaconess Medical Center, for the perioperative management of buprenorphine. The objectives of these guidelines are to address the potential difficulty in achieving analgesia in patients who are taking buprenorphine and present for elective or emergent surgery and to offer guidance for achieving appropriate perioperative pain management. The recommendations in these guidelines are based on the pharmacologic properties of buprenorphine.

In either of the treatment strategies (perioperative discontinuation or maintenance of baseline buprenorphine), for a patient who is maintained on buprenorphine and has a planned surgery, the risk of medication complications is worrisome. Patients who continue to take buprenorphine perioperatively require exceedingly high doses of supplemental opioid but are at risk for inadequate analgesia because of competitive buprenorphine binding. Moreover, high doses of required breakthrough narcotic can lead to overdose and respiratory depression. Clinicians are thus challenged to find an effective balance between pain control and minimized risk of drug complications.

Opioid-dependent patients who bridge to traditional opioids are at an increased risk of opioid abuse; unopposed 


\section{Preoperatively: Assess anticipated postoperative pain and opioid requirements.}

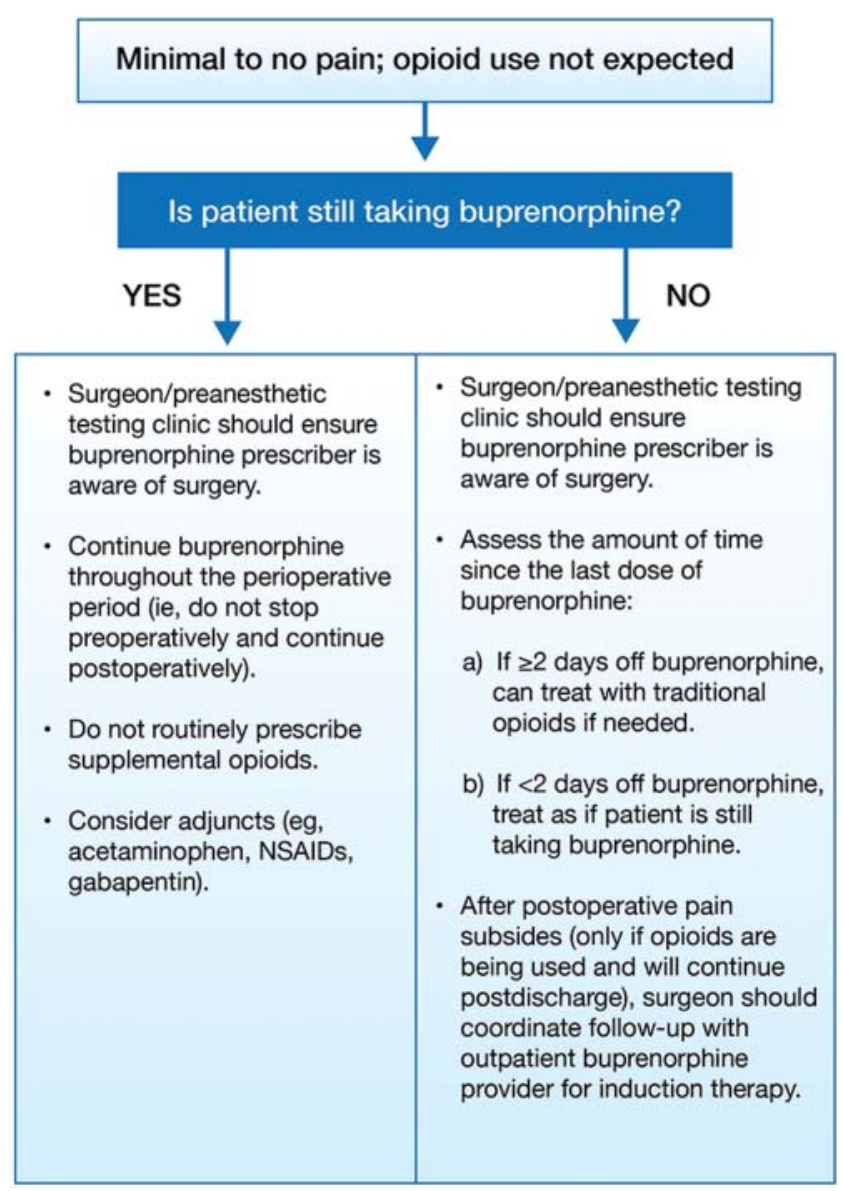

\begin{tabular}{|c|c|}
\hline \multicolumn{2}{|c|}{ Moderate to severe pain; opioid use expected } \\
\hline Is patient still takin & buprenorphine? \\
\hline YES & NO \\
\hline $\begin{array}{l}\text { If evaluated prior to the day of } \\
\text { surgery } \\
\text { - Surgeon/preanesthetic } \\
\text { testing clinic should ensure } \\
\text { buprenorphine prescriber is } \\
\text { aware of surgery and } \\
\text { collaborate with him/her } \\
\text { about the need for bridging } \\
\text { with a pure agonist. } \\
\text { - Patient should not take } \\
\text { buprenorphine for } 2 \text { days } \\
\text { before the day of surgery or } \\
\text { on the day of surgery. } \\
\text { If evaluated on the day of } \\
\text { surgery: } \\
\text { **Strongly consider } \\
\text { postponing surgery.** } \\
\text { - Surgical team should have } \\
\text { patient return to the } \\
\text { buprenorphine prescriber. }\end{array}$ & $\begin{array}{l}\text { - Surgeon/preanesthetic testing } \\
\text { clinic should ensure } \\
\text { buprenorphine prescriber is } \\
\text { aware of surgery. } \\
\text { - Assess the amount of time } \\
\text { since the last dose of } \\
\text { buprenorphine: } \\
\text { a) If } \geq 2 \text { days off buprenorphine, } \\
\text { can treat with traditional } \\
\text { opioids. Patient's course may } \\
\text { be similar to an } \\
\text { opioid-tolerant patient. } \\
\text { b) If <2 days off } \\
\text { buprenorphine, treat as if } \\
\text { patient is still taking } \\
\text { buprenorphine. } \\
\text { After postoperative pain } \\
\text { subsides (if opioids will } \\
\text { continue postdischarge), } \\
\text { surgery team should coordinate } \\
\text { follow-up with outpatient } \\
\text { buprenorphine provider. }\end{array}$ \\
\hline
\end{tabular}

Figure 1. Management of patients on buprenorphine in the perioperative setting for elective surgery. Used with permission of the authors Dr Julie Petro and Dr Cyrus Yazdi.

mu receptor binding can lead to euphoric effects and precipitate relapse. In cases of emergent surgery and trauma, careful planning and management are often not possible, making pain relief particularly challenging. Moreover, patients with opioid use disorder frequently demonstrate a decreased tolerance to pain and increased tolerance to opioid medications, necessitating higher doses and a more frequent dosing schedule. While these risks can often be effectively mitigated in the inpatient setting, they are magnified and problematic during the transition to outpatient postoperative recovery. The primary care clinician is thus faced with the new challenges of drug misuse, relapse, and overmedication after the surgical patient has been discharged home.

Although the focus on the complexity of perioperative buprenorphine management has increased recently, focus specifically on the postoperative management of patients on buprenorphine maintenance therapy following hospital discharge is lacking. With the increasing use of buprenorphine in the treatment of high-risk opioid-dependent patients, the management of postoperative pain in the outpatient setting will become an ever more prevalent challenge in this patient population. While discontinuation of buprenorphine is recommended for some patients, the discussion of appropriate discharge plans and protocols to safely reinduce maintenance buprenorphine therapy - while also managing postoperative pain -is limited. Patients who discontinue buprenorphine, as well as their providers, must be acutely cognizant of the risk of relapse and overdose. ${ }^{4}$ Patients who are maintained on buprenorphine throughout the surgical period may receive a prescription for high-dose oral opioid pain medication. If the patient self-discontinues the buprenorphine after discharge and takes the prescribed opioids, the risk of overdose is high, as the partial agonism of the high dose supplemental opiate is no longer present.

Regardless of the approach taken for perioperative management of buprenorphine maintenance therapy, close follow-up and counseling by the primary buprenorphine prescriber are important for the safe care of these patients. Decisions regarding perioperative buprenorphine management must be made in close concordance with the patient's buprenorphine provider prior to initiation and upon inpatient discharge. Close communication is of the utmost importance; thus, a strong bridge of communication should exist between the perioperative team electing to temporarily discontinue or maintain buprenorphine and the primary outpatient prescriber who will ultimately have the responsibility for safely managing and restarting maintenance therapy while having to factor in acute postoperative surgical pain. Communication includes not only contacting an inpatient addiction specialist 


\section{Preoperatively: Assess anticipated postoperative pain and opioid requirements.}

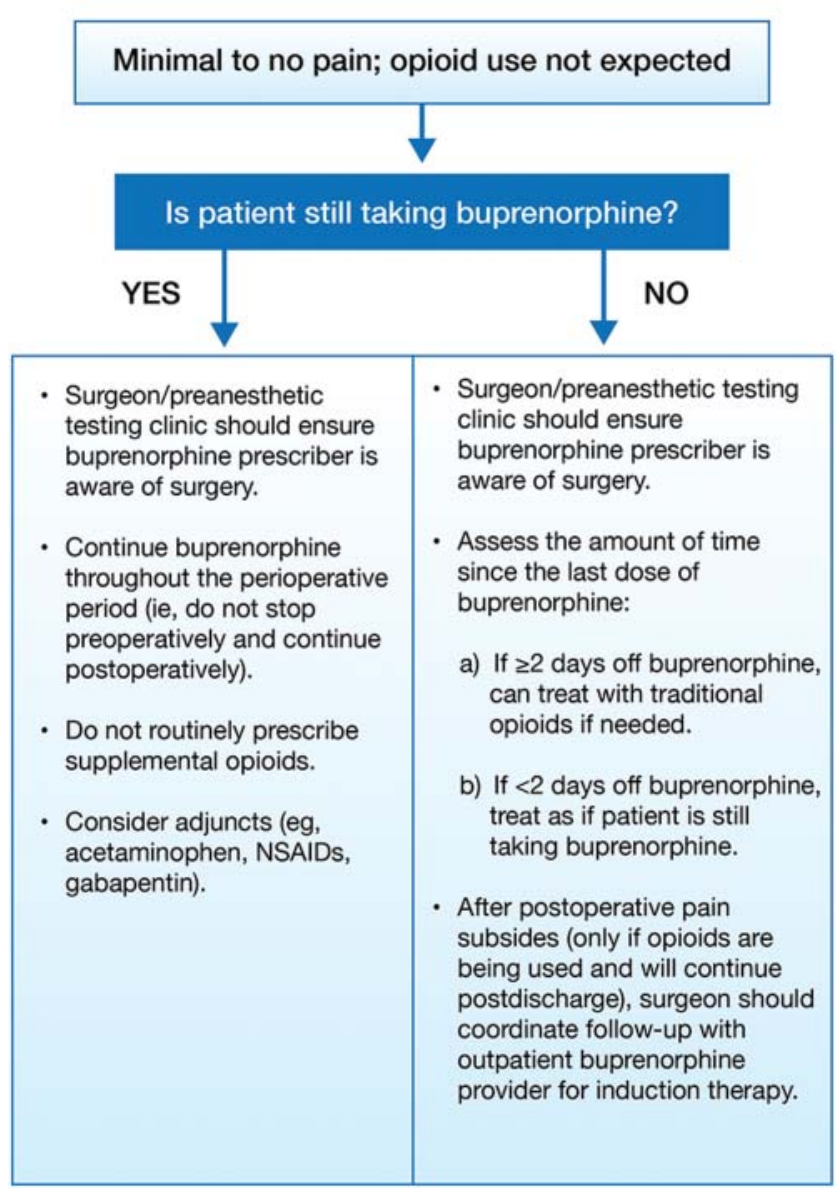

Figure 2. Management of patients on buprenorphine in the perioperative setting for emergent surgery. Used with permission of the authors Dr Julie Petro and Dr Cyrus Yazdi.

during the hospitalization but also providing documentation of the pain regimen prescribed during hospitalization and reviewing the medications for postoperative pain.

Upon reinitiation of care after discharge, the addiction specialist may request random urine drug screens, and if nonbuprenorphine opioids are found in the urine, the patient's addiction/opioid contract may be affected. Furthermore, depending on postoperative prescriptions, the addiction specialist may modify the buprenorphine dose or regimen. In the care of these patients, the importance of bridging the gap between perioperative care and the primary care physician cannot be overstated.

As guidelines for the postoperative outpatient management of buprenorphine therapy are lacking, a consensus statement is needed among expert outpatient primary care physicians, pain medicine specialists, addictionologists, anesthesiologists, and surgeons who all care for this patient population to formalize recommendations that address the questions of both perioperative and postdischarge buprenorphine management and subsequent resumption in the outpatient setting.

\section{ACKNOWLEDGMENTS}

The author has no financial or proprietary interest in the subject matter of this article.

\section{REFERENCES}

1. Rosenthal RN, Goradia VV. Advances in the delivery of buprenorphine for opioid dependence. Drug Des Devel Ther. 2017 Aug 28;11:2493-2505. doi: 10.2147/DDDT.S72543.

2. Jonan $A B$, Kaye $A D$, Urman RD. Buprenorphine formulations: clinical best practice strategies recommendations for perioperative management of patients undergoing surgical or interventional pain procedures. Pain Physician. 2018 Jan;21(1):E1E12.

3. Anderson TA, Quaye ANA, Ward EN, Wilens TE, Hilliard PE, Brummett CM. To stop or not, that is the question: acute pain management for the patient on chronic buprenorphine. Anesthesiology. 2017 Jun;126(6):1180-1186. doi: 10.1097/ ALN.0000000000001633.

4. Stromer W, Michaeli K, Sandner-Kiesling A. Perioperative pain therapy in opioid abuse. Eur J Anaesthesiol. 2013 Feb;30(2): 55-64. doi: 10.1097/EJA.0b013e32835b822b. 